\title{
Adaptive Tuning of Sliding Mode-PID Control in Free Floating Space Manipulator by Sliding Cloud Theory
}

\author{
B. Kharabian, H. Bolandi, A. F. Ehyaei, S. K. Mousavi Mashhadi, S. M. Smailzadeh \\ Department of Electrical Engineering, Iran University of Science and Technology, Tehran, Iran
}

Email address:

Beh16819@yahoo.com (B. Kharabian)

\section{To cite this article:}

B. Kharabian, H. Bolandi, A. F. Ehyaei, S. K. Mousavi Mashhadi, S. M. Smailzadeh. Adaptive Tuning of Sliding Mode-PID Control in Free Floating Space Manipulator by Sliding Cloud Theory. American Journal of Mechanical and Industrial Engineering.

Vol. 2, No. 2, 2017, pp. 64-71. doi: 10.11648/j.ajmie.20170202.12

Received: September 20, 2016; Accepted: December 27, 2016; Published: January 20, 2017

\begin{abstract}
In this paper, a combinational control approach of sliding mode-PID is utilized in free floating space manipulator. Due to float base, nonlinearity of free floating space manipulator dynamics lead to having unstable behaviour and complicated dynamic model. To simplify the dynamic equation, dynamically equivalent manipulator is utilized which is a fixed base manipulator. In this approach, an unique map transfers dynamic parameters of free floating space manipulator to a fixed base manipulator. This transfer enlarges uncertainty bound of parameters. Hence, sliding mode-PID control as a combinational robust control approach is applied in the system. In this case, PID like sliding surface is proposed instead of conventional sliding surface. Adaptive tuning of sliding surface provides a Sliding Cloud that is a kind of dynamic sliding surface. In this way, inclination of system to stable behaviour increases and robustness of system, in the presence of uncertainties, is bolstered.
\end{abstract}

Keywords: Space Manipulator, Free Floating, DEM, Sliding Mod-PID, Adaptive Tuning, Sliding Surface, Sliding Cloud

\section{Introduction}

Space manipulators have significant role in space crafts and perform some services such as maintenance and repair [1]. Therefore, need to control the space manipulators is important challenge. In order to control the base of space manipulator, that is satellite, two major approaches are utilized. First one is free flying approach using reaction wheels. By use of the reaction wheels, power consumption increases. Hence, free floating approach has been proposed to eliminate free flying approach's defects [2]. In this approach reaction wheels are not used and the base moves freely. Free base leads to having complicated dynamic model representation. Therefore, Liong, Xu and Bergerman [3] proposed dynamically equivalent manipulator (DEM) model to work out dynamic model of space manipulator with ease. DEM is a fixed base manipulator with spherical joint as first joint. In this approach, an unique map provides a relation between parameters of space manipulator and corresponding parameters in DEM. Therefore, dynamic model of space manipulator is linearly parameterized [4]. Using this map, the uncertainty bounds of parameters are enlarged. Hence, seeking for capable robust approach in order to handle uncertainty is important issue. Parlaktuno and Ozkan [5] applied an adaptive control method to space manipulator using DEM model. In this approach, unknown parameters such as mass and inertia tensor parameters are estimated by adaptive methods. Shin and Lee [6] proposed an adaptive control in joint space. They used an off-line adaptive identification scheme for these dynamics. In their method, the measurement of the vehicle acceleration is not required and parameters are estimated accurately using persistently exciting torque signals. $\mathrm{Gu}$ and $\mathrm{Xu}$ [7] proposed an extended robot model in order to solve this nonlinear parameterization problem.

In this paper, sliding mode-PID with PID like sliding surface is proposed to reinforce robustness of system in the presence of uncertainties. Conventional sliding surface forms a line( two dimensional) in phase plane but PID like sliding surface provides a surface(three dimensional) in phase plane. Therefore, an extra alternative is provided to vindicate the stability conditions. Furthermore, in order to achieve perfect performance in the system, adaptive tuning of PID like sliding surface is posed that creates a Sliding Cloud. Sliding Cloud is an intelligent dynamic sliding surface moving onto dynamics of system. In this case, a time varying Sliding Cloud is moved by adaptive tuning of sliding surface coefficients to incline 
sliding surface to system dynamics. In other words, on the one hand control law propels dynamics of system to sliding surface, and on the other hand, Sliding Cloud moves onto the dynamics. As a result, this method renders a reinforced tendency to stability situation. Kharabian [8] used fuzzy logic to tune PID like sliding surface. In this paper, the proposed approach has less complicacy; as a result, the implementation of that would be more convenient. In sliding mode control, uncertainty bound of system should be calculated to determine limit of constant coefficient of sliding mode control law $(k)$ which is difficult process. Consequently, fuzzy logic is used to tune this constant gain eventuating in having simple calculation and control law.

This paper is organized as follows. In section 2, the dynamic model of free floating space manipulator is represented. Sliding mode control with PID like sliding surface is described in section 3. Adaptive tuning of PID like sliding surface is performed in section 4. In section 5, simulation results are shown. Conclusions are rendered in final section.

\section{Dynamic of Free Floating Space Manipulator}

In order to represent dynamic model of space manipulator, free floating approach is selected that leads to decreasing the power consumption. Free floating approach poses a free base space manipulator with no control effort on the base. Hence, nonlinearity of dynamic model is high. The $n$-DOF space manipulator on a free-floating base, as shown in Figure 1, includes n-link manipulator and its base. The base of space manipulator is represented as link 1, the links of the manipulator as links 2 through $\mathrm{n}+1$.

In Figure $1, \quad(\varphi, \theta, \omega)$ are $Z-Y-Z$ Euler angles representating the orientation of the space manipulator's base, $J_{i}$ is the joint connecting $(i-1)$ th link and $i$ th link, $\theta_{i}$ is the rotation of the space manipulator's $i$ th link around joint $J_{i} . L_{i}$ is vector connecting $J_{i}$ to $C_{i}, R_{i}$ is vector connecting $C_{i}$ to $J_{i+1}$. Assuming no external forces and torques act on the space manipulator, its centre of mass $C_{o}$ remains fixed in inertial space and can be selected as the origin of the inertial coordinate frame. Dynamic equation of space manipulator is obtained as

$$
M(q) \ddot{q}+C(q, \dot{q}) \dot{q}=\tau
$$

where $M(q) \in R^{(n+3) \times(n+3)}$ is inertia matrix $C(q \cdot \dot{q}) \dot{q} \in R^{(n+3)}$ is vector of the Coriolis and centrifugal forces, $\tau$ is torque acting upon the joints of the space manipulator.

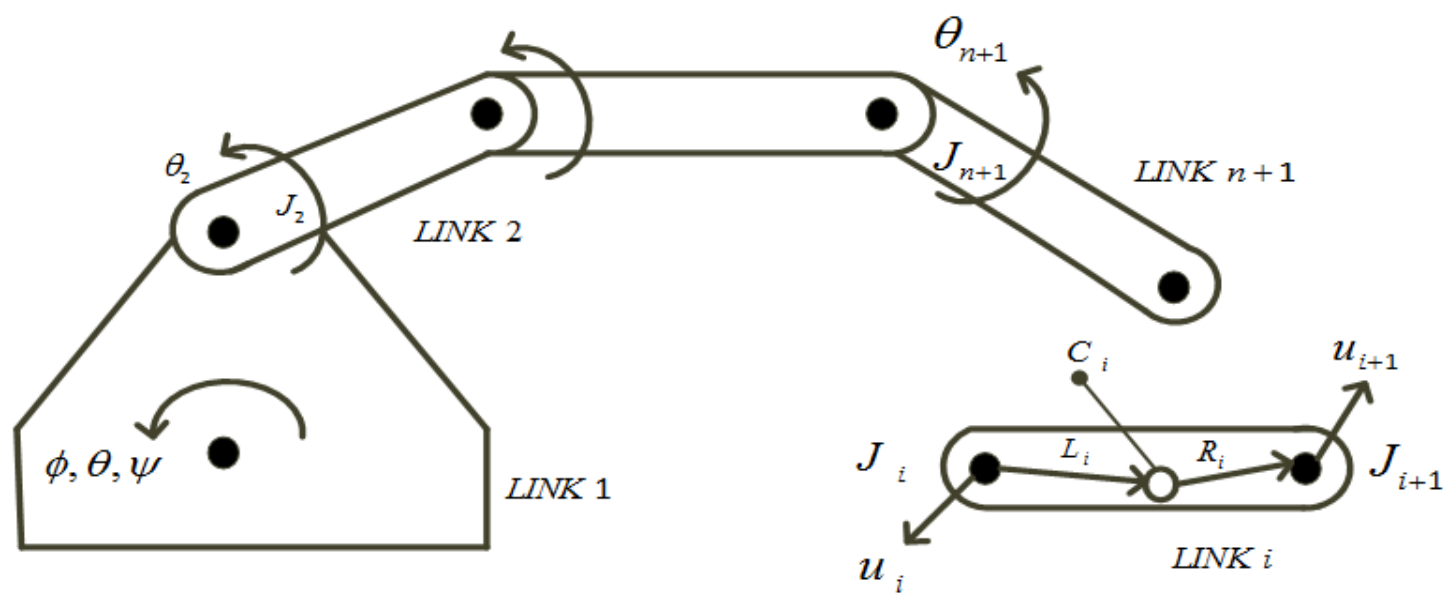

Figure 1. Scheme of free-floating space manipulator.

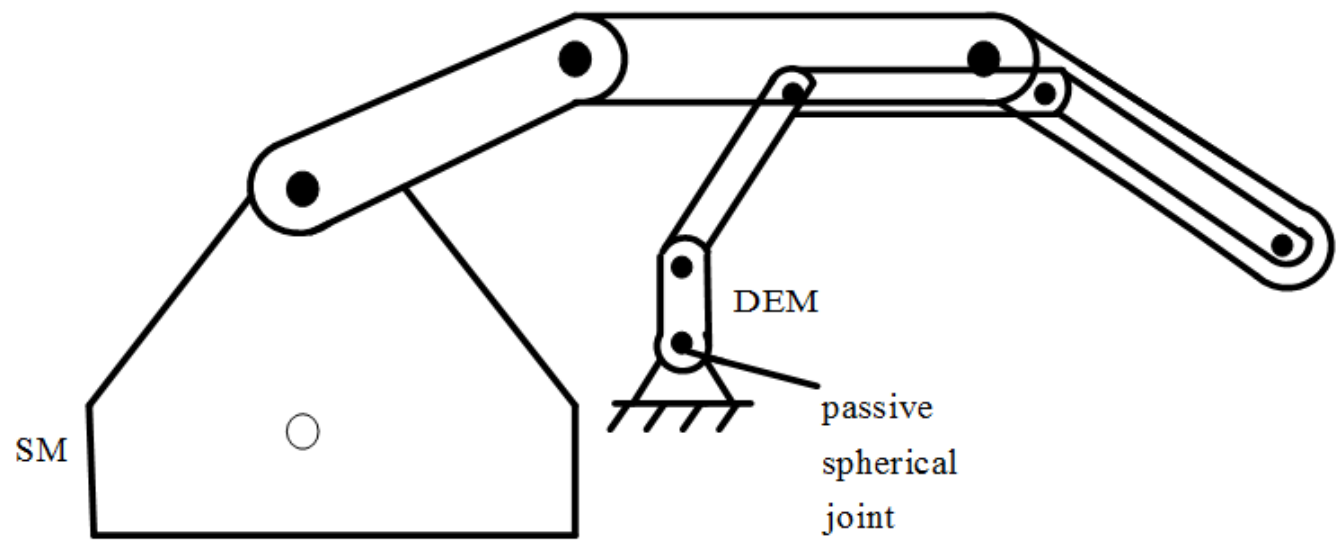

Figure 2. Space manipulator and its corresponding DEM. 
Dynamical equivalent manipulator renders a fixed base with passive spherical joint as first joint. In this case, inertia parameters of space manipulator map to the DEM. Thereby, dynamic model of manipulator will be linearly parameterized. In Figure 2, the axis of the space manipulator's $i$ th joint is parallel to the axis of the DEM's $i$ th joint. The displacement of each of the DEM's joints during motion is identical to the displacement of the corresponding space manipulator joint. As in Figure 3 is shown, in DEM coordinate frames are respectively attached to the centre of mass of the DEM links. In Figure 3, $\left(\varphi^{\prime}, \theta^{\prime}, \omega^{\prime}\right)$ are $Z-Y-Z$ Euler angles representing the orientation of the DEM's passive spherical joint, $J_{i}^{\prime}$ is the joint connecting the DEM's $(i+1)$ th link and $i$ th link, $\theta_{i}^{\prime}$ is the relative rotation of the DEM's link around joint $J_{i,}^{\prime}, l_{c i}$ is the vector connecting $J_{i}^{\prime}$ to $C_{i}^{\prime}, \omega_{i}^{\prime}$ is the angular velocity of $C^{\prime}$.

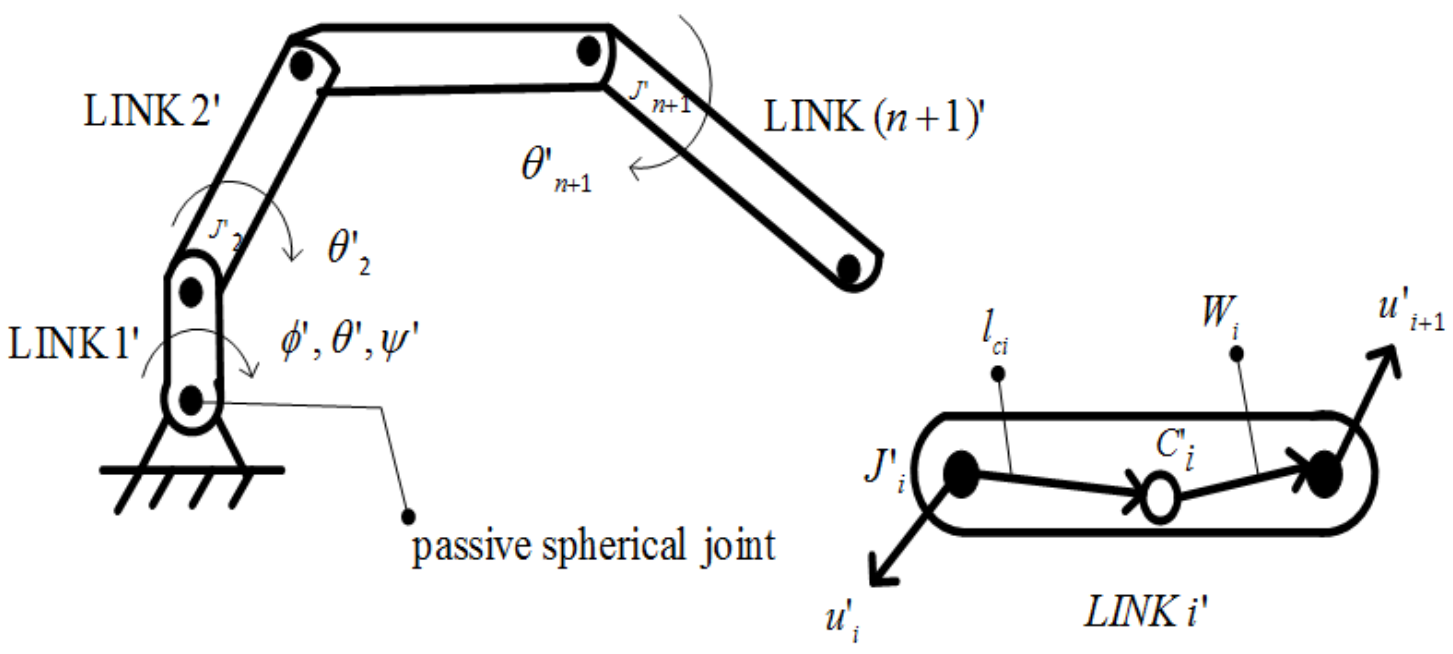

Figure 3. Fixed-base robot manipulator with a passive spherical joint at the base.

This map is shown as below

$$
\begin{gathered}
m_{i}^{\prime}=\frac{m_{i}\left(\sum_{k=1}^{n+1} m_{k}\right)^{2}}{\sum_{k=1}^{i-1} m_{k} \sum_{k=1}^{i} m_{k}}, \quad i=2, \ldots, n+1 \\
I_{i}^{\prime}=I_{i}, \quad i=1, \ldots, n+1, \\
\left.W_{i}=R_{i}\left(\frac{\left.W_{1}=\frac{R_{1} m_{1}}{\sum_{k=1}^{i} m_{k}}\right)}{\sum_{k=1}^{i} m_{k}}\right)+\sum_{k=1}^{\sum_{i}\left(\frac{\sum_{k=1}^{n+1} m_{k}}{n+1} m_{k}\right.}\right), \quad i=2, \ldots, n+1, \\
\sum_{k=1}^{i-1} l_{c_{1}}=0, \\
\sum_{c_{i}=L_{i}\left(\frac{\sum_{k=1}^{n+1} m_{k}}{\sum_{k=1} m_{k}}\right),} \quad i=2, \ldots, n+1
\end{gathered}
$$

Through this map inertial properties of the space manipulator are preserved. In this model, mapping uncertainties including masses $\left(d m_{i}^{\prime}\right)$, links' length $\left(d W_{i}\right)$, location of centre of mass $\left(d l_{c i}\right)$ and inertia tensor $\left(d I_{i}^{\prime}\right)$ due to inaccuracy in $m_{i}, L_{i}, R_{i}$ and $I_{i}$ are considered in the dynamic model of DEM. Therefore, dynamic equation estimation is written as

$$
\hat{M}^{\prime}\left(q^{\prime}\right) \ddot{q}^{\prime}+\hat{C}^{\prime}\left(q^{\prime}, \dot{q}^{\prime}\right) \dot{q}^{\prime}=\tau^{\prime}
$$

The DEM is dynamically equivalent to the space manipulator. In other words, if exerted torque on the space manipulator's joints is equal to exerted torque on the DEM's joints, starting from equal initial condition, DEM's joints and space manipulator's joints track similar paths (i.e. if $q\left(t_{0}\right)=q^{\prime}\left(t_{0}\right)$ and $\left.q(t)=q^{\prime}(t)\right)$. then $\left.q(t)=q^{\prime}(t)\right)$.

\section{Sliding Mode-Pid Control}

Using DEM approach leads to enlarging uncertainty bounds of parameters. Therefore, sliding mode control as a robust control approach is utilized to stabilize system in spite of large uncertainty bound. Conventional sliding surface is given as

$$
\begin{aligned}
& S=K_{D} e^{i}+K_{P} e^{\prime} \\
& \left(e^{\prime}=q^{\prime}{ }_{d}-q^{\prime}\right)
\end{aligned}
$$

that is two dimensional. Control law is designed to propel the dynamics of system to sliding surface (where $S=0$ ). Phase 
plane of sliding surface is as Figure 4.

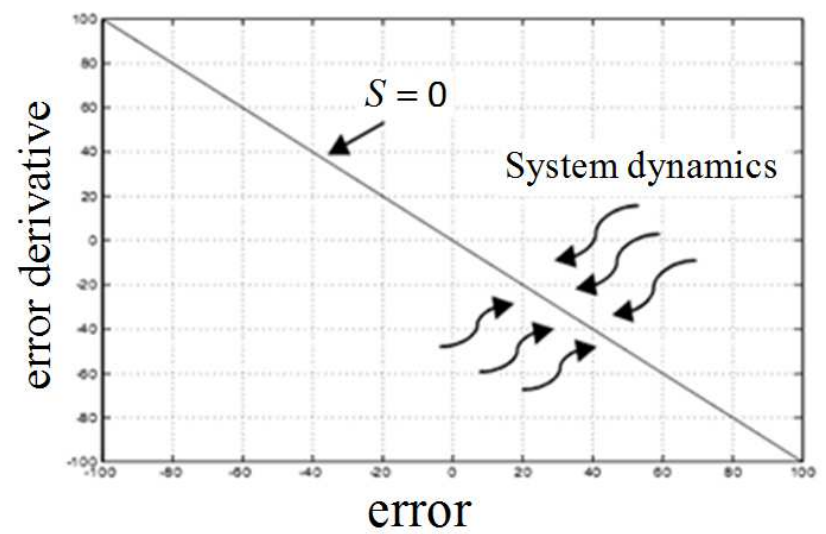

Figure 4. Two dimensional sliding surface.

In order to modify performance of the system and develop more robust system, sliding surface is represented as PIDlike rendering one more degree of freedom. In this way more alternatives ( $K_{I} \int e^{\prime} d t$ renders one more degree of freedom) are provided to vindicate lyapunov condition that leads to stability $(S=0)$. The PID like sliding surface is given as

$$
\begin{aligned}
& S=K_{D} e^{\prime}+K_{P} e^{\prime}+K_{I} \int \dot{e}^{\prime} d t \\
& \left(e^{\prime}=q_{d}^{\prime}-q^{\prime}\right)
\end{aligned}
$$

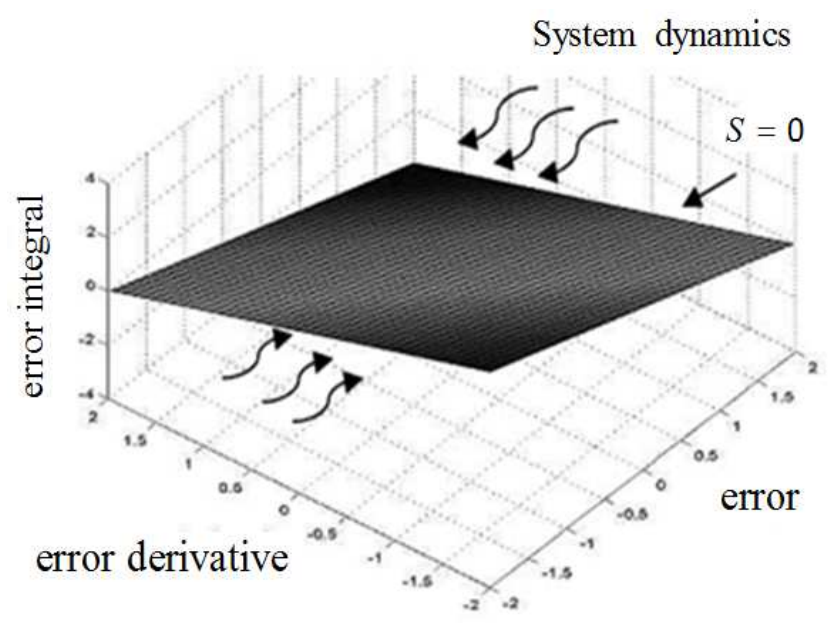

Figure 5. Three dimensional (PID like) sliding surface.

Phase plane of PID like sliding surface is shown in Figure 5. In this case, it can be expected to have a little more robust behaviour than two dimensional sliding surface (line was replaced with surface).

According to the sliding condition, control law is selected as

$$
\tau^{\prime}=S-k \operatorname{sgn}(S)
$$

where sliding condition is as below

$$
\frac{1}{2} \frac{\mathrm{d}}{\mathrm{dt}}\left(\mathrm{S}^{\mathrm{T}} \mathrm{S}\right) \leq-\eta|\mathrm{S}|
$$

In order to get rid of chattering phenomenon $\operatorname{sat}(\mathrm{S})$ is used instead of $\operatorname{sgn}(\mathrm{S}) . \operatorname{sat}(\mathrm{S})$ is defined as

$$
\operatorname{sat}\left(\frac{S}{\delta}\right)= \begin{cases}1, & \frac{S}{\delta} \geq 1 \\ \frac{S}{\delta}, & -1 \leq \frac{S}{\delta} \leq 1 \\ -1, & \frac{S}{\delta} \leq-1\end{cases}
$$

where $\delta$ is boundary layer.

In conventional sliding mode control, $k$ is determined by limit of overall uncertainty bound of system. According to the sliding condition, $k$ is considered as

$$
k(i, i) \geq\left|\Delta f_{i}\right|
$$

where $\Delta f_{i}$ is overall uncertainty bound of system.

In this paper, fuzzy logic is used to tune $k$ based on the values of error, error derivative, and integral of error which form $S$.

Membership functions of error, error derivative, integral of error and $k$ are shown in Figures 6, 7, 8 and 9.

As with (6), follows up the changes of $S$. Therefore, the following fuzzy rules are considered to adjust $k$.

- If $e$ is small and $\dot{e}$ is Nsmall and $\int e d t$ is small then $k$ is small

- If $e$ is small, $\dot{e}$ are Nsmall and $\int e d t$ is medium then $k$ is small

- If $e$ is small and, is Nsmall and $\int e d t$ is big then $k$ is small

- If $e$ is medium and, $\dot{e}$ is small and $\int e d t$ is small then $k$ is small

- If $e$ is big and, $\dot{e}$ is Nsmall and $\int e d t$ is small then $k$ is small

- If $e$ is small, $\dot{e}$ is Nmedium and $\int e d t$ is medium then $k$ is medium

- If $e$ is medium, $\dot{e}$ is Nmedium and $\int e d t$ is medium then $k$ is medium

- If $e$ is big, $\dot{e}$ is Nmedium and $\int e d t$ is medium then $k$ is big

- If $e$ is medium, $\dot{e}$ is Nmedium and $\int e d t$ is big then $k$ is big

- If $e$ is medium, $\dot{e}$ is Nbig and $\int e d t$ is medium then $k$ is big

- If $e$ is big, $\dot{e}$ is Nbig and $\int e d t$ is big then is big

- If $e$ is small, $\dot{e}$ is Nsmall and $\int e d t$ is big then $k$ 
is medium

- If $e$ is medium, $\dot{e}$ is Nmedium and $\int e d t$ is small then $k$ is big

Lyapunov function for system is proposed as

$$
v=\frac{1}{2} S^{T} S
$$

According to lyapunov conditions, stability of system is proved. Lyapunov conditions are given as

$$
\begin{gathered}
v=\frac{1}{2} S^{T} S>0 \\
\dot{v}=\frac{d}{d t}\left(\frac{1}{2} S^{T} S\right)=S^{T} \dot{S} \leq 0
\end{gathered}
$$

Rewriting dynamic model of system in the base of sliding surface, following equation is obtained.

$$
\hat{M}^{\prime} \dot{S}+\hat{C}^{\prime} S=Y-K_{D} \tau^{\prime}
$$

where

$$
\begin{aligned}
& Y=\hat{M}^{\prime}\left(K_{D} \ddot{q}_{d}^{\prime}+\dot{K}_{D} \dot{e}+K_{P} \dot{e}+\dot{K}_{P} e^{\prime}+K_{I} e^{\prime}+\dot{K}_{I} \int \dot{e} d t\right) \\
& +\hat{C}^{\prime}\left(K_{D} \dot{q}_{d}+K_{P} \dot{e}^{\prime}+K_{I} \int \dot{e}^{\prime} d t\right)
\end{aligned}
$$

Substituting 13 into 12 , obtains

$$
\dot{v}=-S^{T} \hat{M}^{-1} \hat{C} S+S^{T} \hat{M}^{-1}\left(Y-K_{D} \tau^{\prime}\right)
$$

And substituting (6) into (13), obtains

$$
\dot{v}=-S^{T} \hat{M}^{-1} \hat{C} S+S^{T} \hat{M}^{-1}\left(Y-K_{D}\left(S-k \text { sat }\left(\frac{S}{\delta}\right)\right)\right)
$$

where $K_{P}, K_{I}$ and $K_{D}$ that are constant are selected so that

$$
\dot{v} \leq-\mu \sum_{i=1}^{n+1}\left|S_{i}\right| \leq 0
$$

where $\mu$ is a positive constant.

\section{Adaptive Sliding Mode-PID Control}

By using PID like sliding surface with constant gains, performance of system is not efficient. On the other hand, utilization of high control gains causes increment of oscillations. Hence, the gains of sliding surface are tuned adaptively to ascertain proper performance with desired result. The gains are tuned adaptively as

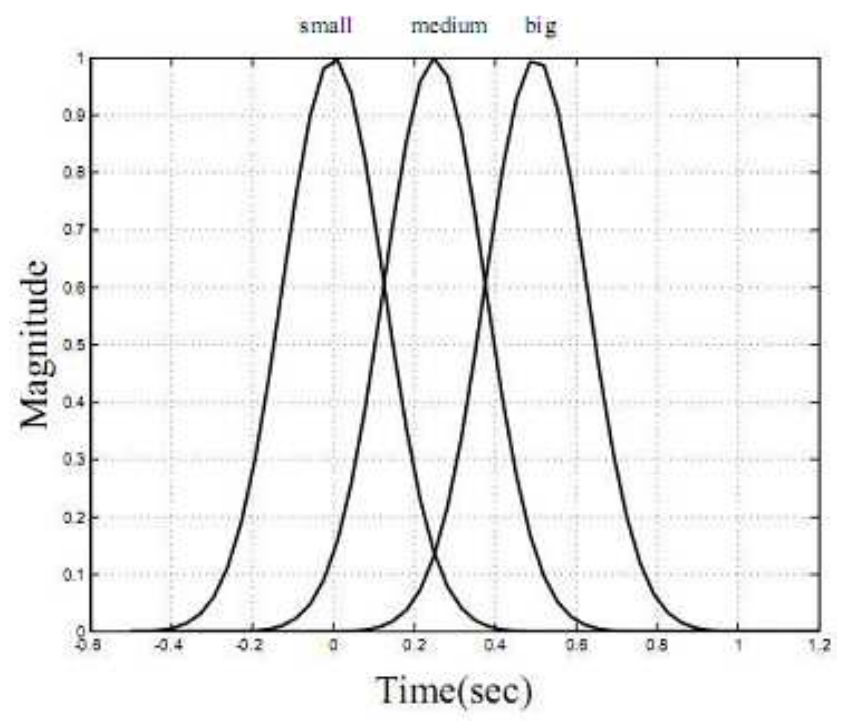

Figure 6. Membership functions of error.

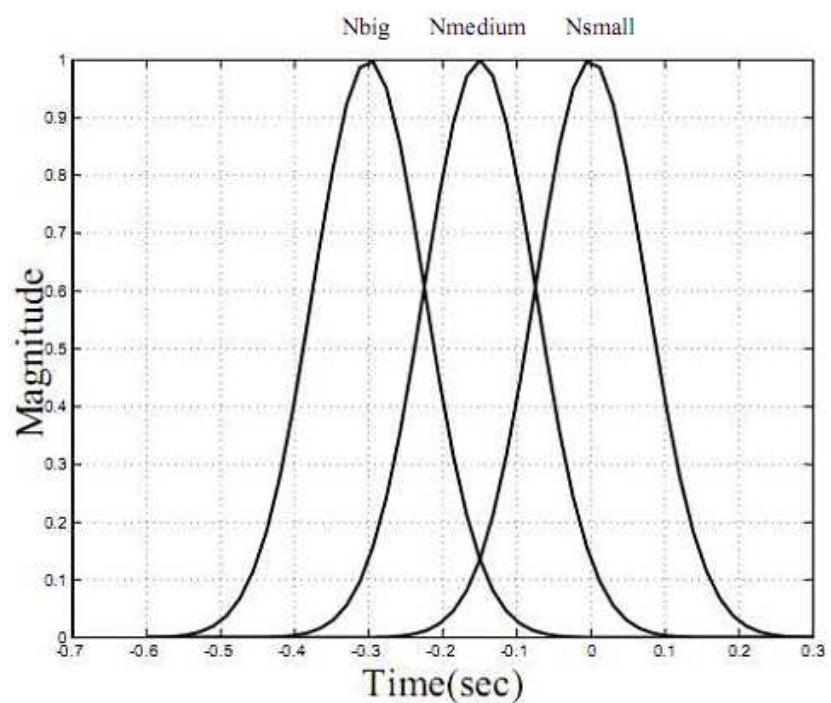

Figure 7. Membership functions of error derivative.

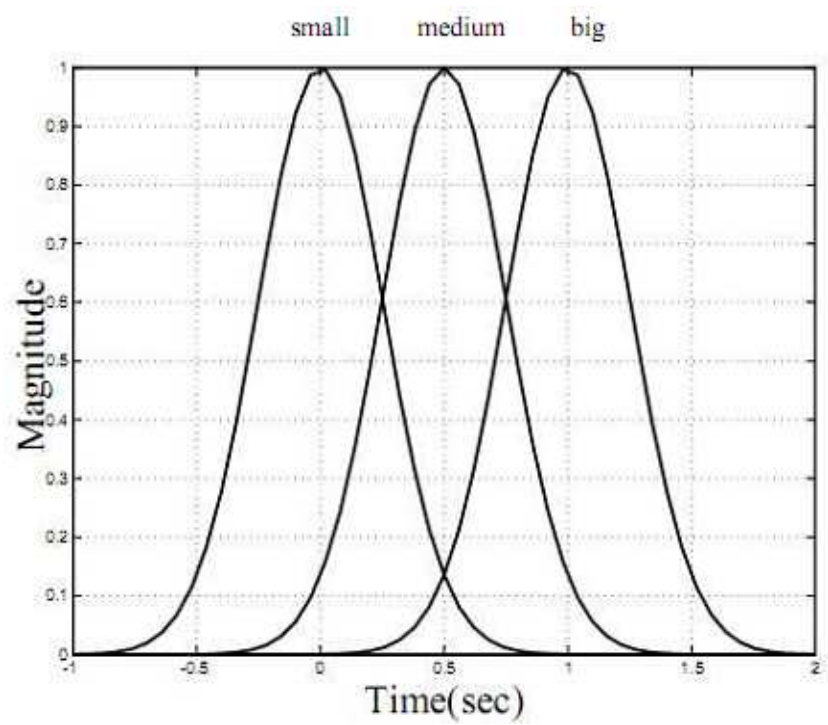

Figure 8. Membership functions of integral of error. 


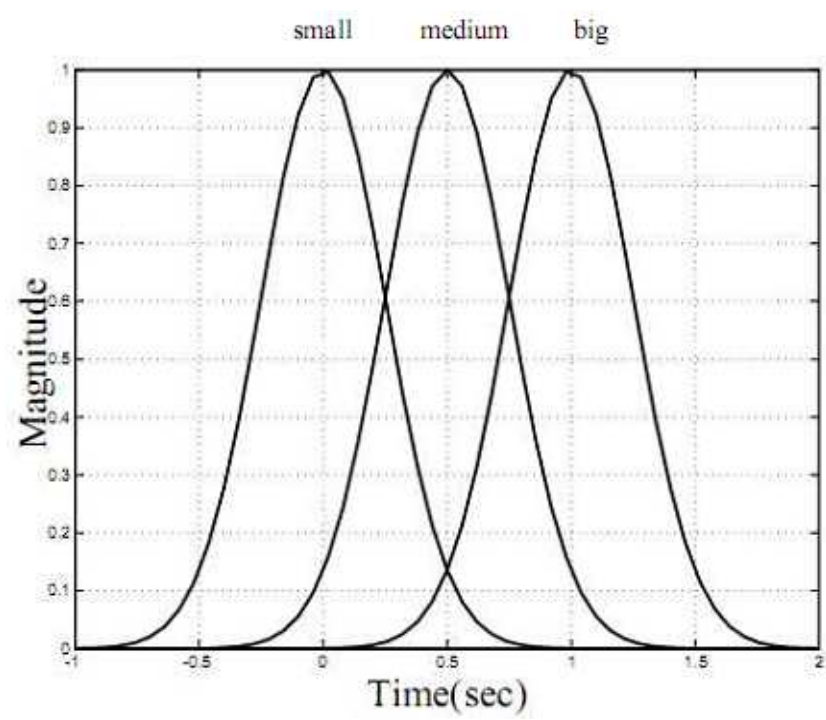

Figure 9. Membership functions of $k$

$$
\begin{aligned}
& K_{P_{2}}=\dot{e} 2, K_{D_{2}}=\int e_{2} d t, K_{I_{2}}=e_{2} \\
& K_{P_{3}}=e_{3}, K_{D_{3}}=\int e_{3} d t, K_{I_{3}}=e_{3}
\end{aligned}
$$

Therefore, sliding surface is as below

$$
S=e e+e \int e d t+e \int e d t
$$

that is a time varying sliding surface. Time varying coefficients of sliding surface make a dynamic Sliding Cloud, as Figure 10, which has abundant inclination to system dynamics. Hence, sliding surface and system dynamics reach to each other with ease. This means that system has great tendency to stability and shows desired performance.

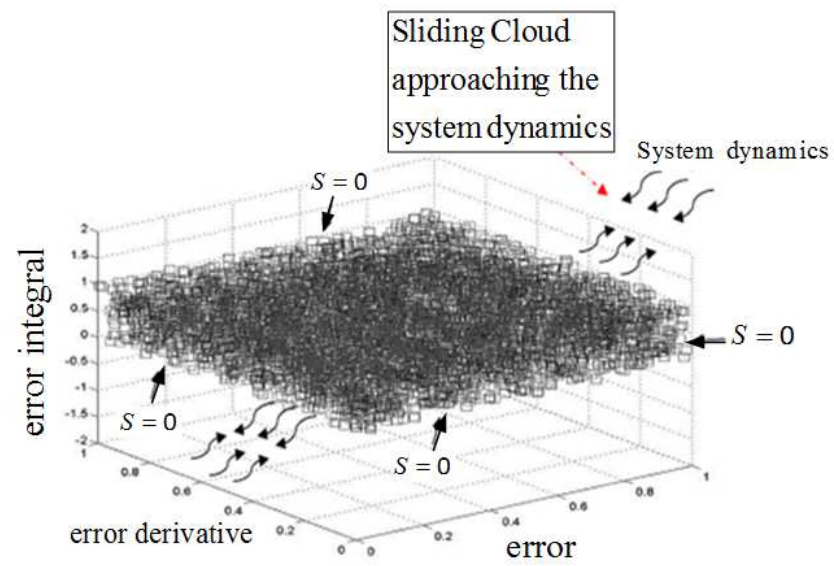

Figure 10. Three dimensional ( PID like) Sliding Cloud.

In sense of lyapunov, Sliding Cloud provides more flexibility to vindicate the lyapunov condition. Lyapunov function with exerted adaptively tuned gains is obtained as

$$
v=\frac{1}{2} S^{T} S=\frac{1}{2}\left(\dot{e} e+\dot{e} \int e d t+e \int e d t\right)^{T}\left(\dot{e} e+\dot{e} \int e d t+e \int e d t\right)
$$

\section{Simulation Results}

In this section, a 2 DOF space manipulator with free base is considered as system. By using the map, fixed base manipulator with 3 DOF is obtained, so that first joint is a passive spherical joint. Hence, trajectories of joints 2 and 3 are considered as significant results. Nominal parameters of space manipulator and DEM are presented in Tables 1 and 2, respectively.

As in Figures 11 and 12 are shown performance of system is not sufficiently acceptable. Therefore, Sliding Cloud is utilized to achieve desired behaviour.

In order to tune PID like sliding surface gains, selected adaptive gains as (18) are inserted in the sliding surface. Outcomes are shown in Figures. 13 and 14 (desired trajectory is $\left.q_{\text {desired }}=0.5+0.2 \sin (0.1 t)\right)$.

Table 1. Nominal parameters of space manipulator.

\begin{tabular}{lllll}
\hline link & $\mathbf{L}_{\mathbf{i}}(\mathbf{m})$ & $\mathbf{R}_{\mathbf{i}}(\mathbf{m})$ & $\mathbf{m}_{\mathbf{i}}(\mathbf{k g})$ & $\mathbf{I}_{\mathbf{i}}\left(\mathbf{k g . m ^ { 2 }}\right)$ \\
\hline base & - & 0.5 & 4 & 0.4 \\
2 & 0.5 & 0.5 & 1 & 0.1 \\
3 & 0.5 & 0.5 & 1 & 0.1 \\
\hline
\end{tabular}

Table 2. Nominal parameters of DEM.

\begin{tabular}{lllll}
\hline link & $\boldsymbol{W}_{\boldsymbol{i}}(\boldsymbol{m})$ & $\boldsymbol{l}_{\boldsymbol{c i}}(\boldsymbol{m})$ & $\boldsymbol{m}_{\boldsymbol{i}}(\mathbf{k g})$ & $\boldsymbol{I}_{\boldsymbol{i}}\left(\mathbf{k g} \cdot \boldsymbol{m}^{2}\right)$ \\
\hline 1 & 0.333 & 0 & 4 & 0.4 \\
2 & 0.75 & 0.333 & 1.8 & 0.1 \\
3 & 0.917 & 0.417 & 1.2 & 0.1 \\
\hline
\end{tabular}

Dynamical representation of 2 DOF space manipulator is given as

$$
M(q) \ddot{q}+C(q, \dot{q}) \dot{q}=\left[\begin{array}{c}
0 \\
\tau_{2} \\
\tau_{3}
\end{array}\right]
$$

By using PID like (three dimensional) with constant gains, following outcomes for joint trajectory of DEM, shown in Figures 11 and 12, are obtained (desired trajectory are selected as $\left.q_{\text {desired }}=0.5+0.2 \sin (0.1 t)\right)$.

$$
\begin{aligned}
K_{P} & =\left[\begin{array}{ccc}
0.1 & 0 & 0 \\
0 & 0.4 & 0 \\
0 & 0 & 0.4
\end{array}\right], \mathrm{K}_{\mathrm{D}}=\left[\begin{array}{ccc}
0.1 & 0 & 0 \\
0 & 14 & 0 \\
0 & 0 & 15
\end{array}\right], \\
K_{I} & =\left[\begin{array}{ccc}
0.1 & 0 & 0 \\
0 & 3 & 0 \\
0 & 0 & 4
\end{array}\right], \mathrm{k}=\left[\begin{array}{lll}
1 & 0 & 0 \\
0 & 1 & 0 \\
0 & 0 & 1
\end{array}\right] .
\end{aligned}
$$

As in Figures 11 and 12 are shown performance of system is not sufficiently acceptable. Therefore, Sliding Cloud is utilized to achieve desired behaviour.

In order to tune PID like sliding surface gains, selected adaptive gains as (18) are inserted in the sliding surface. Outcomes are shown in Figures 13 and 14 (desired trajectory is $\left.q_{\text {desired }}=0.5+0.2 \sin (0.1 t)\right)$. 


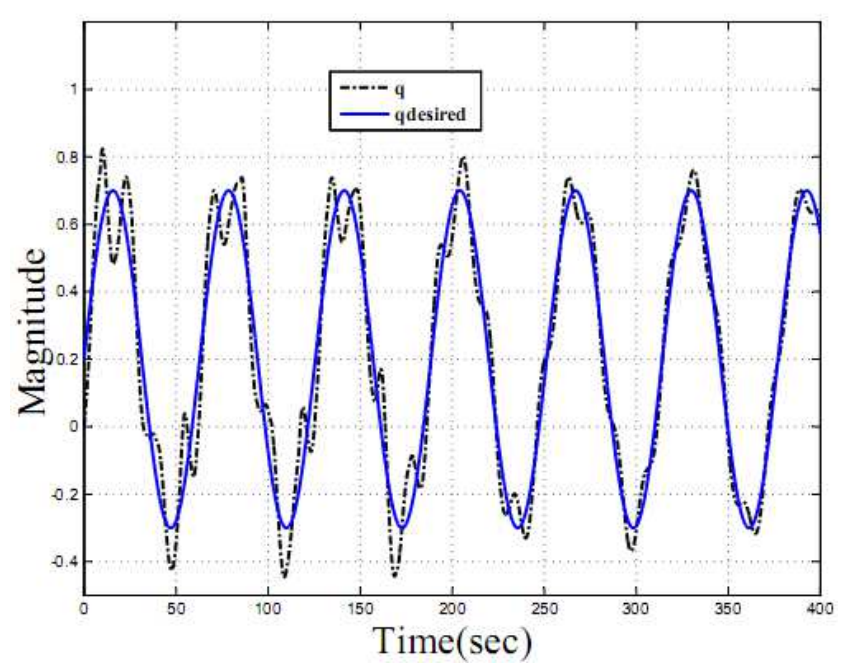

Figure 11. Trajectory of joint 2 with PID like (three dimensional) sliding surface.

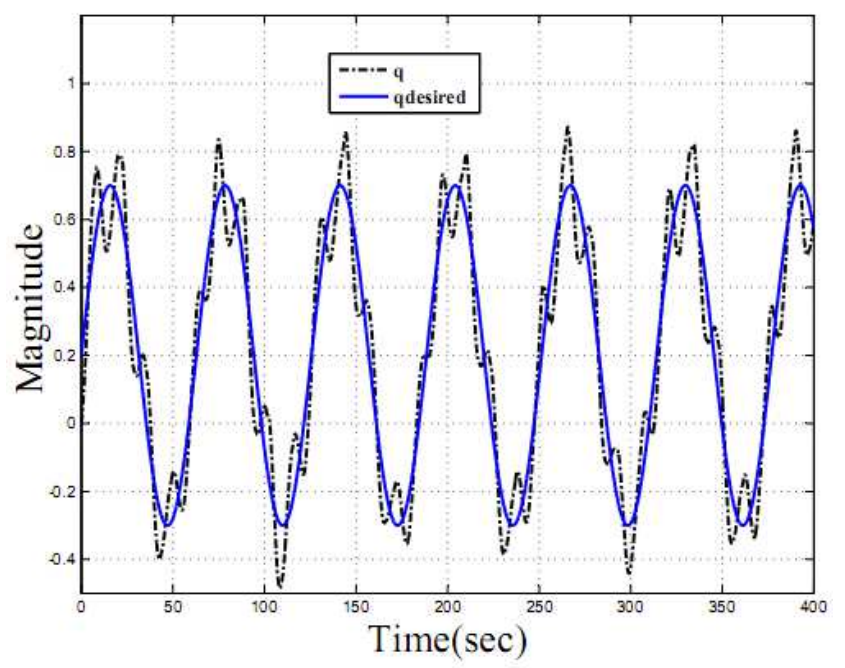

Figure 12. Trajectory of joint 2 with PID like (three dimensional) sliding surface.

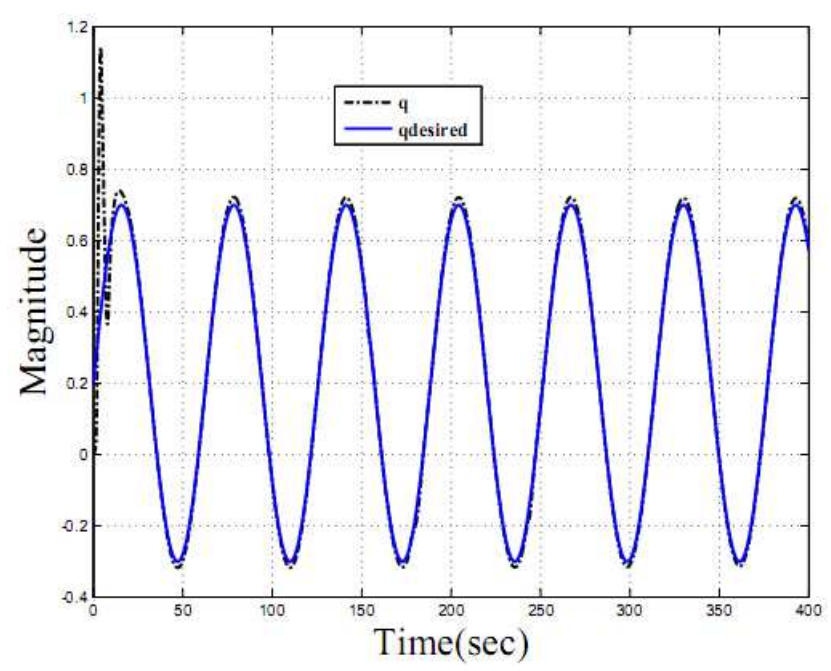

Figure 13. Trajectory of joint 2 with adaptive gains for sliding surface.

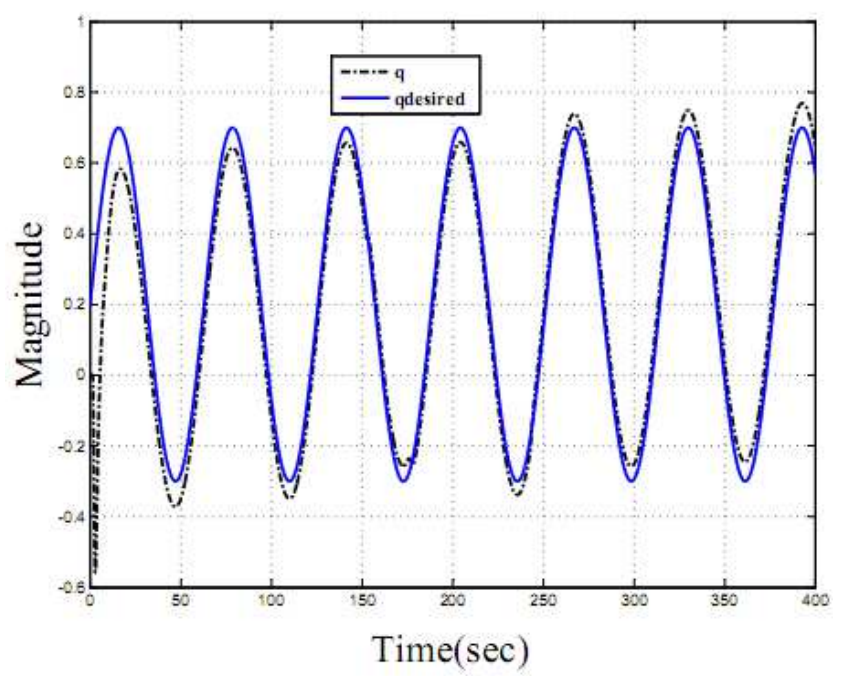

Figure 14. Trajectory of joint 3 with adaptive gains for sliding.

\section{Conclusion}

In this paper, adaptive sliding mode-PID was exerted in free floating space manipulator. In Order to represent dynamic model of space manipulator, dynamical equivalent manipulator (DEM) was used to simplify model representation. Mapping parameter of free floating space manipulator onto DEM involves enlargement of uncertainty bound. Therefore, sliding mode control is used to handle the system with regard to uncertainties. In order to get to more degrees of freedom, PID like sliding surface is proposed. To have a more acceptable performance for system, coefficients of sliding surface were tuned and time varying sliding surface is provided named Sliding Cloud. Sliding Cloud lead to having abundant inclination to propel sliding surface and dynamics system to each other. Simulation results show proficiency of this control approach.

\section{References}

[1] C. Wang, B. Feng, G. Ma and C. Ma, "Robust Tracking Control of Space Robots Using Fuzzy Neural Network", IEEE International Symposium on Computational Intellig-ence in Robotics and Automation, pp. 181-185, 2005.

[2] G. Legnani, B. Zappa, R. Adamini and F. Casolo, "A contribution to dynamics of free-flying space manipulator", echanical and Machine Theory, Vol. 34, pp. 359-372, 1999.

[3] B. Liang, Y. Xu and M. Bergerman, "Mapping a space manipulator to a dynamically equivalent manipulator", ASME Journal of Dynamic Systems, Measurement and Control, Vol. 120, pp. 1-7, 1998.

[4] E. Papadopoulos and S. Dubowsky, "On the nature of control algorithms for free-floating space manipulators", IEEE Trans. on Robotics and Automation, Vol. 7, pp. 750-758, 1991.

[5] O. Parlaktuna and M. Ozkan, "Adaptive control of freefloating space manipulators using dynamically equivalent manipulator model", Robotics and Autonomous Systems, Vol. 46, 185-193, 2004. 
[6] J. H. Shin and J. J. Lee, "Dynamic control with adaptive identification for free-flying space robots in joint space", Robotica, pp. 12541-551, 1994.

[7] Y. L. Gu and Y. Xu, "A normal from augmentation approach to adaptive control of space robot systems", Proceedings of the IEEE International Conference on Robotics and Automation, Vol. 2, pp. 731-737, 1993.

[8] B. Kharabian, "Fuzzy Sliding Mode-PID Control for Space Manipulator Using Dynamically Equivalent Manipulator Model", International Journal of Control and Automation Vol. 7, pp. 143-158, 2014.

[9] H. Okubo, T. Matsudo and N. Katsuda, "Reduction of Control Toque for Space Manipulators Using Dynamic Manipulability Ellipsoid”, International Symposium on Artificial Intelligence, Robotics and Automation in Space, Japan, 2003.

[10] T. W. Yang, Z. Q. Sun, S. K. TSO and W. L. Xu, “Traje-ctory Control of a Flexible Space Manipulator Utilizing a MacroMicro Architecture", IEEE International Confer-ence of Robotics \& Automation, Vol. 2, pp. 2522 - 2528, 2003.

[11] Y. Xu, H. Y. Shum, T. Wade and J. J. Lee, "Parameterization Control of Space and Adaptive Robot Systems", IEEE Trans. on Aerospace and Electronic Systems, Vol. 30, pp. 435-451, 1994.

[12] Y. Fenga, X. Yu, and Z. Manc, "non-singular terminal sliding mode control of manipulators", Automatica, Vol. 38, pp. 21592167,2002
[13] A. Rhif, "A High Order Sliding Mode Control with PID Sliding Surface: Simulation on a Torped",. International Journal of Information Technology, Control and Automation (IJITCA), Vol. 2, pp. 13-18, 2012.

[14] T. H. Tran, Q. P. Ha and H. T. Nguyen, "Robust Non Overshoot Time Responses Using Cascade Sliding Mode-PID Control", Journal of Advanced Computational Intelligence, Vol. 11, pp. 1224-1231, 2007.

[15] V. Parra-Vega, S. Arimoto, Y. H. Liu, G. Hirzinger and P. Akella, "Dynamic Sliding PID Control for Tracking of Robot Manipulator: Theory and Experiments", IEEE Trans. On Robotics and Automation, Vol. 19, pp. 967-976, 2003.

[16] S. Dubowsky and V. Vafa, "On the Dynamics of manipula-tors in Space Using The Virtual Manipulator", Approach. Proceeding of the 1987 IEEE International Conference on Robotics and Automation, pp. 579-585, 1987.

[17] S. Abiko and G. Hirzinger, "Computational Efficient Algorithms for Operational Space Formulation of Branching Arms on a Space Robot", IEEE/RSJ Int. Conference on Intelligent Robots and Systems, Acropolis Convention Center Nice, France, 2008.

[18] M. Fallahi and S. Azadi, "Robust Control of Dc Motor Using Fuzzy Sliding mode Control with PID Compensator", Proceeding of the International Multi Conference of Engineers and Computer Scientists, Vol. 2, 2009. 